\title{
SPAG6 and L1TD1 are transcriptionally regulated by DNA methylation in non-small cell lung cancers
}

\author{
Corinna Altenberger ${ }^{1,2}$ (D), Gerwin Heller ${ }^{1,2}$, Barbara Ziegler ${ }^{1,2}$, Erwin Tomasich ${ }^{1,2}$, Maximilian Marhold ${ }^{1,2}$, \\ Thais Topakian 1,2, Leonhard Müllauer ${ }^{2,3}$, Petra Heffeter ${ }^{2,4}$, György Lang ${ }^{2,5,7}$, Adelheid End-Pfützenreuter ${ }^{2,5}$, \\ Balazs Döme ${ }^{2,5,6,7}$, Britt-Madeleine Arns ${ }^{8}$, Walter Klepetko ${ }^{2,5}$, Christoph C. Zielinski ${ }^{1,2}$ and Sabine Zöchbauer-Müller ${ }^{1,2^{*}}$
}

\begin{abstract}
Background: DNA methylation regulates together with other epigenetic mechanisms the transcriptional activity of genes and is involved in the pathogenesis of malignant diseases including lung cancer. In non-small cell lung cancer (NSCLC) various tumor suppressor genes are already known to be tumor-specifically methylated. However, from the vast majority of a large number of genes which were identified to be tumor-specifically methylated, tumor-specific methylation was unknown so far. Thus, the major aim of this study was to investigate in detail the mechanism(s) responsible for transcriptional regulation of the genes SPAG6 and L1TD1 in NSCLCS.
\end{abstract}

Methods: We analysed publically available RNA-sequencing data and performed gene expression analyses by RTPCR. DNA methylation analyses were done by methylation-sensitive high-resolution melt analyses and bisulfite genomic sequencing. We additionally investigated protein expression using immunohistochemistry. Cell culture experiments included tumor cell growth, proliferation, viability as well as colony formation assays. Moreover, we performed xenograft experiments using immunodeficient mice.

Results: We observed frequent downregulation of SPAG6 and L1TD1 mRNA expression in primary tumor (TU) samples compared to corresponding non-malignant lung tissue (NL) samples of NSCLC patients. We furthermore observed

re-expression of both genes after treatment with epigenetically active drugs in most NSCLC cell lines with downregulated SPAG6 and L1TD1 mRNA expression. Frequent tumor-specific DNA methylation of SPAG6 and L1TD1 was detected when we analysed TU and corresponding NL samples of NSCLC patients. ROC curve analyses demonstrated that methylation of both genes is able to distinguish between TU and NL samples of these patients. Immunohistochemistry revealed a close association between SPAG6/L1TD1 methylation and downregulated protein expression of these genes. Moreover, by performing functional assays we observed reduced cell growth, proliferation and viability of pCMV6-L1TD1 transfected NSCLC cells. In addition, reduced volumes of tumors derived from pCMV6L1TD1 compared to pCMV6-ENTRY transfected NCI-H1975 cells were seen in a xenograft tumor model.

Conclusions: Overall, our results demonstrate that SPAG6 and L1TD1 are tumor-specifically methylated in NSCLCs and that DNA methylation is involved in the transcriptional regulation of these genes. Moreover, in vitro as well as in vivo experiments revealed tumor-cell growth suppressing properties of L1TD1 in NSCLC cells.

Keywords: Epigenetics, DNA methylation, Non-small cell lung cancer, SPAG6, L1TD1

\footnotetext{
* Correspondence: sabine.zoechbauer-mueller@meduniwien.ac.at

${ }^{1}$ Department of Medicine I, Clinical Division of Oncology, Medical University

of Vienna, Währinger Gürtel 18-20, 1090 Vienna, Austria

${ }^{2}$ Comprehensive Cancer Center, Medical University of Vienna, Vienna, Austria

Full list of author information is available at the end of the article
} 


\section{Background}

DNA methylation (referred to as methylation) is a major epigenetic modification which regulates gene expression mainly by binding methyl-CpG binding proteins (MBDs) and their associated chromatin remodeling factors to DNA $[1,2]$. It has been shown that methylation plays an important role in various molecular and cellular processes including embryonic development and genomic imprinting as well as in the pathogenesis of malignant diseases [3-7]. Methylation occurs by the covalent addition of a methyl group to the $5^{\prime}$ carbon of cytosines within CpG dinucleotides which is catalysed by DNA methyltransferases (DNMTs) [8, 9]. Unlike genetic changes, methylation may be reversed by DNMT inhibitors such as 5-aza-2'-deoxycytidine (5-AzadC) and 5azacytidine (5-AzaC). A synergistic effect on re-expression of by methylation silenced genes by DNMT inhibitors in combination with histone deacetylase inhibitors like trichostatin A (TSA) was reported [10].

In non-small cell lung cancers (NSCLCs) numerous tumor suppressor genes (TSGs) are already known which are frequently methylated. Besides other epigenetic and genetic factors methylation may be responsible for transcriptional inactivation of these genes [11-15]. However, from the vast majority of $\sim 500$ genes which were identified to be tumor-specifically methylated in a genome-wide approach, tumor-specific methylation was unknown so far [12]. When we additionally analysed publically available microarray data in primary tumors (TU) compared to non-malignant lung tissue (NL) samples of NSCLC patients, we observed tumor-specifically downregulated expression of many of these genes [11, 16, 17]. An extensive PubMed search revealed that many of these genes are functionally uncharacterised in NSCLCs and in other cancer types. Based on all these observations, we selected the genes SPAG6 (Sperm Associated Antigen 6) and L1TD1 (LINE-1 Type Transposase Domain Containing 1) for detailed investigation. SPAG6 is located in the chromosomal region 10p12.2 and is thought to be a cancer-testis antigen (CTA) [18]. CTAs represent a large family of cancer-associated antigens which are expressed in immunoprivileged tissues such as testis but were also detected in tumor tissues of various origins including lung cancer [19]. SPAG6 is also expressed in normal lung tissues where it is associated with ciliary function [20]. It encodes a microtubuleassociated protein which either functions as microtubule itself or binds to microtubules to form the cytoskeleton of the cell (www.pantherdb.org). There is increasing evidence that the expression of CTAs might be involved in tumorigenesis, however, so far there are no reports available about an involvement of SPAG6 in malignant disease biology or cancer cell invasiveness [21]. L1TD1 is located in the chromosomal region $1 \mathrm{p} 31.3$ where frequent loss of heterozygosity $(\mathrm{LOH})$ was observed in NSCLCs [22]. This gene encodes a stem-cell specific RNA-binding protein required for self-renewal of human embryonic stem cells and for cancer cell proliferation [23]. Since the mechanism(s) of inactivation of both, SPAG6 and L1TD1, were not studied in detail and their role in the pathogenesis of NSCLCs was unclear so far, we were interested to further investigate these genes.

Thus, we determined gene expression, methylation and re-expression of SPAG6 and L1TD1 in various NSCLC cell lines to elucidate if methylation is associated with the transcriptional inactivation of these genes. Moreover, we investigated tumor-specific methylation of these genes in a large number of NSCLC patients and compared these data as well as mRNA expression data with clinico-pathological characteristics of NSCLC patients. We also analysed protein expression of both genes in a subset of NSCLC patients and compared these results with SPAG6 and L1TD1 methylation. In addition, potential tumor-cell growth suppressing properties of these genes were investigated in in vitro studies and, for L1TD1, also in in vivo xenograft experiments.

Overall, we identified methylation as a mechanism involved in the regulation of transcriptional activity of SPAG6 and L1TD1 in NSCLCs. Furthermore, our results indicate that L1TD1 functions as a tumor cell growth suppressor in NSCLC cells.

\section{Methods}

\section{Publically available databases}

IlluminaHiSeq RNA-sequencing (RNA-seq) data were obtained from "The Cancer Genome Atlas" (TCGA) database (https://cancergenome.nih.gov), Cancer Browser (https://genome-cancer.ucsc.edu) and from cBioPortal for Cancer Genomics (http://www.cbioportal.org) [24-28]. For analyses of single nucleotide variants (SNVs) and deletions of SPAG6 and L1TD1 lung adenocarcinoma (LUAD) and lung squamous cell carcinoma (LUSC) datasets were used. A summary of the clinico-pathological data of analysed patients is shown in Additional file 1: Table S2. For additional mRNA expression analyses, breast invasive carcinoma (BRCA), colon and rectum adenocarcinoma (COADREAD), head and neck squamous cell carcinoma (HNSC), kidney clear cell carcinoma (KIRC), liver hepatocellular carcinoma (LIHC) and prostate adenocarcinoma (PRAD) datasets were used (https://cancergenome.nih.gov). Data visualization of SNVs and homozygous and heterozygous deletions was performed using Caleydo software [29].

\section{Tumor cell lines and tissue samples}

NSCLC cell lines A549 and NCI-H1993 were purchased from the American Type Culture Collection (ATCC), cell lines HCC827, NCI-H1650 and NCI-H1975 were 
kindly provided by Dr. Walter Berger (Institute of Cancer Research, Medical University of Vienna, Austria). Cell lines were maintained and grown as described [11]. Detailed descriptions of NSCLC and non-lung cancer cell lines are provided in Additional file 1: Table S1 and Table S4. Normal human bronchial epithelial cell (NHBECs) pellets were purchased from Promocell. For re-expression experiments tumor cells were treated either with the demethylating agent 5-AzadC alone or with a combination of 5-AzadC and the HDAC inhibitor TSA as described [30]. Untreated cells were used as controls. In addition, DNA of breast cancer (MCF-7, MDAMB-453, MDA-MB-468, MDA-MB-231, BT20), colon cancer (HCT-15, HT29), ovarian cancer (SK-OV3, A2780), pancreatic cancer (AsPC-1, BxPC-3) as well as head and neck cancer cell lines (CAL27, FaDu) were provided by various members of the Medical University of Vienna, Austria. Primary TU and corresponding NL samples of 146 stage I-III NSCLC patients who underwent surgical resection of the tumor were collected and stored in liquid nitrogen until use. From 97 of these patients clinico-pathological characteristics including gender, age, histology, tumor stage, lymph node stage, disease stage, disease free survival (DFS) and overall survival (OS) were available. Moreover, from 35 of these patients formalin-fixed, paraffin embedded (FFPE) TU and NL samples were available and were used for immunohistochemistry. This study was approved by the local ethics committee.

\section{Methylation-sensitive high resolution melting analysis (MS-HRM)}

Genomic DNA from NHBECs, 18 tumor cell lines and tissue samples of 146 NSCLC patients was isolated and modified by treatment with sodium bisulfite using EpiTect Bisulfite kit (Qiagen) [11]. The genomic sequences of SPAG6 and L1TD1 were obtained from ENSEMBL database (release 69). Primers were designed using Methyl Primer Express v1.0 software and are listed in the Additional file 1: Table S3. MS-HRM analyses were performed using a Rotor-Gene Q cycler (Qiagen) [31].

\section{Bisulfite genomic sequencing (BGS)}

Sodium bisulfite treated genomic DNA was amplified (primer sequences listed in Additional file 1: Table S3). PCR products containing sequences which were also analysed by MS-HRM analyses were cloned using TOPO $^{\circ}$ TA Cloning ${ }^{\circ}$ Kit for Sequencing (Invitrogen). Four clones per cell line were sequenced using M13 primers.

\section{Real-time reverse transcription PCR (RT-PCR)}

Total RNA was isolated from NHBECs and from 5 NSCLC cell lines and reverse transcribed using
OmniScript Reverse Transcriptase Kit (Qiagen) [11]. Expression of SPAG6 was determined using Taqman Gene Expression Assays Hs00542625_m1 (SPAG6) and Hs03929097_g1 (GAPDH) in a StepOne cycler (Applied Biosystems) and expression of L1TD1 was determined using Qiagen's QuantiTect ${ }^{\circ}$ SYBR Green PCR Kit (primer sequences see Additional file 1: Table S3). The $\Delta \Delta \mathrm{Ct}$ method was used to calculate differences in gene expression [32].

\section{Immunohistochemistry (IHC)}

Tissue microarrays with $1 \mathrm{~mm}$ cores and $5 \mu \mathrm{m}$ sections from FFPE TU and NL samples were used for protein expression analyses of SPAG6 and L1TD1. Samples were stained with the rabbit polyclonal antibodies HPA038440 (SPAG6 1:100, Sigma Aldrich) and HPA028501 (L1TD1 1:100, Sigma Aldrich). Results of IHC were scored as no staining (-), weak staining $(-/+)$, moderate staining $(1+)$ or strong staining $(\geq 2+)$. For comparison with methylation results patients whose TU showed no or weak staining were grouped as "negative by IHC" while patients whose TU showed moderate or strong staining were grouped as "positive by IHC" as reported previously [12].

\section{Cell transfection}

NCI-H1975 cells were transfected with pCMV6-ENTRY (PS100001, Origene) and pCMV6-L1TD1 (RC219014, Origene) expression vectors using Lipofectamine ${ }^{\circ}$ LTX reagent (Invitrogen). Additionally, a pCMV6-GFP vector was constructed by subcloning the GFP coding sequence into existing pCMV6-ENTRY vector (primer sequences see Additional file 1: Table S3). Stably transfected NCIH1975 cells were selected by G418 treatment (Invitrogen) and transfection efficacy was analysed by RT-PCR and Western blotting [11].

\section{Immunoprecipitation and Western blot (IP-WB)}

IP was used to enrich L1TD1 protein after transfection with pCMV6-L1TD1 expression vector of NCI-H1975 cells. Transfected cells were lysed in Pierce IP Lysis Buffer (Thermo Scientific) containing PhosSTOP and EDTA-free inhibitors (Roche). IP was carried out using L1TD1 Antibody (HPA028501, Sigma-Aldrich), normal rabbit IgG (\#2729, Cell Signaling) and Protein A/G PLUS-Agarose beads (Santa Cruz). Western blot analysis was performed as described [11].

\section{Cell proliferation assay}

The xCELLigence Real-Time Cellular Analysis (RTCA) System (Roche) was used to measure cell proliferation in real-time. Stably transfected NCI-H1975 cells were seeded in triplicates and cell proliferation was monitored [11]. 


\section{Cell viability assay}

Stably transfected NCI-H1975 cells were seeded in triplicates in 96-well plates and incubated with CellTiter ${ }^{\text {тs }}$ Blue reagent (Promega). Fluorescence was measured repeatedly using a TriStar microplate reader (Berthold Technologies) [11].

\section{Colony formation assay}

After selection of stably transfected NCI-H1975 cells, cells were grown until differences in colony-forming abilities were detected. Before imaging cells were stained with $0.05 \%$ crystal violet dye.

\section{Xenograft tumor model}

All animal experiments were approved by and carried out according to guidelines from the Austrian Federal Ministry of Science, Research and Economy and the Animal Ethics Committee of the Medical University of Vienna. For the xenograft tumor model female NSG JAX (NOD.Cg-Prkdc $\left.c^{\text {scid }} I l 2 r g^{t m 1 W j l} / S z J\right)$ mice (NSG mice) were purchased from Charles River Laboratories and housed under pathogen-free conditions in the animal facility of the Medical University of Vienna. $5 \times 10^{5}$ NCIH1975 cells stably transfected with L1TD1 expression vector or with empty control vector were injected subcutaneously into the left respectively right flanks of 4 17 weeks old NSG mice. Animals were checked for overall health regularly and tumor size was measured using a digital caliper. Tumor volume was calculated using the standard formula (length $\mathrm{x}$ width ${ }^{2}$ )/2 [33]. At the end of the experiment mice were sacrificed by cervical dislocation and dissected tumors were snap frozen and processed for RT-PCR analyses.

\section{Statistical analyses}

Wilcoxon signed rank tests and receiver operating characteristic (ROC) curve analyses were performed to determine methylation differences between $\mathrm{TU}$ and $\mathrm{NL}$ samples obtained by MS-HRM analyses. Spearman correlation was determined to investigate correlation between methylation and expression data. To calculate statistical differences between the tumor volumes of xenografts two-way ANOVA test was performed. These analyses were performed using GraphPad Prism 6 software with two-sided p-values $<0.05$ considered as statistically significant. MS-HRM data were compared with clinico-pathological characteristics of NSCLC patients. $\mathrm{Chi}^{2}$ tests/ Fisher's exact tests were applied to calculate differences between groups and t-tests were used to calculate differences between means. For methylation analyses $\mathrm{T} / \mathrm{N}$ ratios were calculated and a ratio of $>1.5$ was used as cut off for samples to be considered as methylated. Survival analyses of patients were performed using log rank testing. Cox regression was used for univariate analyses on overall survival and to calculate hazard ratios with $95 \%$ confidence intervals. A p-value $<0.05$ was considered as statistically significant. Analyses were performed using the statistics software PASW (version 18). Kaplan-Meier plots were generated using Kaplan-Meier plotter [34]. The cut-off values for "low" and "high" SPAG6 as well as L1TD1 mRNA expression were automatically defined by KM plotter software.

\section{Results}

Downregulation of SPAG6 and L1TD1 mRNA expression in NSCLCs and in other tumor entities

We analysed publically available RNA-seq data from TCGA database to investigate SPAG6 and L1TD1 mRNA expression in NSCLC patients. By comparison of RNA-seq expression values in TU and corresponding NL samples of LUAD and LUSC datasets, we observed a statistically significant downregulation of SPAG6 and L1TD1 mRNA expression in lung adenocarcinomas $(p<0.0001$, respectively; Fig. 1a) as well as in lung squamous cell carcinomas $(p<0.0001$, respectively; Fig. 1b). In addition, we analysed mRNA expression of these genes also in tumors of other histologies by the use of different datasets from the TCGA database [24]. Compared to non-malignant tissues we observed statistically significant downregulation of SPAG6 in
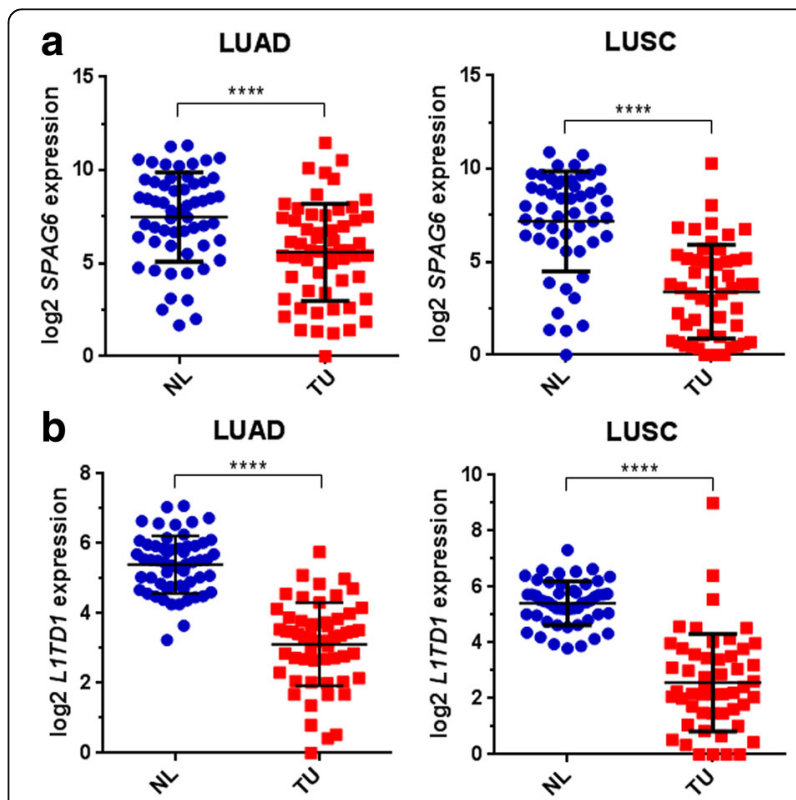

Fig. 1 SPAG6 and LITD1 mRNA expression in TU and corresponding NL samples of NSCLC patients in TCGA datasets. Comparison of SPAG6 $\mathbf{a}$ and L1TD1 $\mathbf{b}$ mRNA expression in TU and NL samples of 57 adenocarcinoma (LUAD dataset) and 51 squamous cell carcinoma patients (LUSC dataset), respectively. Normalized log2 expression values are shown, ${ }^{* * *} p<0.0001$. Only patients with matched TU and NL samples were used for this analysis. TU, primary tumor sample; NL, normal lung tissue sample 
breast carcinomas $(p=0.0039)$, colorectal carcinomas $(p<$ $0.0001)$, head and neck carcinomas $(p=0.0030)$, kidney clear cell carcinomas $(p<0.0001)$ and in hepatocellular carcinomas $(p=0.0046)$, but not in prostate carcinomas (Additional file 2: Figure S1). In contrast, L1TD1 expression was significantly downregulated in breast carcinomas $(p=0.0253)$, colorectal carcinomas $(p=0.0224)$ and in prostate carcinomas $(p<0.0001)$, but not in head and neck carcinomas, kidney clear cell carcinomas and in hepatocellular carcinomas (Additional file 2: Figure S1).

\section{Transcriptional silencing of SPAG6 and L1TD1 in cancer} cell lines is caused by methylation of SPAG6 and L1TD1

We additionally determined mRNA expression of these genes in NSCLC cells as well as in NHBECs. While SPAG6 and L1TD1 mRNA expression was observed in NHBECs, expression of these genes was decreased in cells of all 5 NSCLC cell lines analysed (Fig. 2a and d). In order to investigate if downregulated SPAG6 and L1TD1 expression in NSCLC cells may be caused by methylation, we developed MS-HRM assays to analyse methylation of the $5^{\prime}$ - promoter regions of these genes. SPAG6 and L1TD1 were found to be methylated in cells of all 5 NSCLC cell lines, but not in NHBECs (Fig. 2a and d).

Moreover, we treated cells of the 5 NSCLC cell lines found to be SPAG6 and L1TD1 methylated with either 5-AzadC alone and with a combination of 5-AzadC and TSA. We observed an upregulation of SPAG6 as well as of L1TD1 mRNA expression after treatment with epigenetically active drugs compared to untreated cells by RT-PCR (Fig. 2b and e). To confirm data obtained by MS-HRM analyses, we also performed bisulfite genomic sequencing (BGS) of parts of the 5 '-regions of these genes in cells of selected NSCLC cell lines and in NHBECs. While most of the $56 \mathrm{CpG}$ sites analysed were found to be SPAG6 methylated in A549 (79\%) and in NCI-H1975 (92\%) cells, only a few CpG sites were methylated in NHBECs (4\%, Fig. 2c). Differences in the percentage of SPAG6 methylated CpG sites between NSCLC cells and NHBECs were statistically significant $(p<0.0001)$. L1TD1 was methylated in $77 \%$ and $93 \%$ of 24. CpG sites analysed in A549 and in NCI-H1975 cells, however, in NHBECs only 3\% of CpG sites were methylated for this gene (Fig. 2f). Differences of L1TD1 methylation between NSCLC cell lines and NHBECs were statistically significant $(p<0.0001)$.

Moreover, we determined methylation of SPAG6 and L1TD1 in 5 breast cancer, 2 colon cancer, 2 ovarian cancer, 2 pancreatic cancer as well as 2 head and neck cancer cell lines. All these tumor cell lines were found to be SPAG6 and L1TD1 methylated with percentages of methylation ranging between $71 \%$ (SK-OV3 cells) and 98\% (FaDu cells) for SPAG6 methylation and between
$88 \%$ (BxPC-3 cells) and $100 \%$ (FaDu cells) for L1TD1 methylation (Additional file 1: Table S4).

\section{SPAG6 and L1TD1 are tumor-specifically methylated in NSCLC patients}

We also investigated SPAG6 and L1TD1 methylation in TU and corresponding NL samples of 146 stage I-III NSCLC patients by MS-HRM analyses. Differences in SPAG6 and L1TD1 methylation between TU and NL samples were statistically significant $(p<0.0001$, respectively) demonstrating that both genes are tumor-specifically methylated (Fig. 3a and b). Furthermore, ROC curve analyses of methylation results revealed that SPAG6 as well as L1TD1 methylation is able to distinguish TU from NL samples of NSCLC patients $(p<0.0001$, respectively; Fig. 3a and b). Moreover, for each patient $\mathrm{T} / \mathrm{N}$ ratios of SPAG6 and L1TD1 methylation were calculated as the \% of methylation in the primary $\mathrm{TU} / \%$ of methylation in the corresponding NL sample [12]. Considering patients with a $\mathrm{T} / \mathrm{N}$ ratio $\geq 1.5$ as methylated, $79 \%$ of them were SPAG6 methylated and $81 \%$ of them were $L 1 T D 1$ methylated, respectively. In addition, $\mathrm{T} / \mathrm{N}$ ratios of SPAG6 and L1TD1 methylation were used to compare methylation results with clinico-pathological characteristics of the patients. No statistically significant associations were observed.

However, we observed a statistically significant shorter OS of patients with squamous cell carcinoma subtype and low SPAG6 mRNA expression levels as well as of patients with adenocarcinoma subtype and low L1TD1 mRNA expression levels (Additional file 3: Figure S2; https://cancergenome.nih.gov/).

\section{SPAG6 and L1TD1 SNVs and deletions in NSCLC patients}

To investigate if other mechanisms besides methylation are involved in transcriptional regulation of SPAG6 and L1TD1, we analysed SNVs as well as homozygous and heterozygous deletions in LUAD and LUSC SNP and aCGH datasets of NSCLC patients. In lung adenocarcinoma patients only $3 \%$ had SPAG6 SNVs. While no homozygous SPAG6 deletions were detected, $22 \%$ of these patients showed SPAG6 heterozygous deletions (Additional file 4: Figure S3). In lung squamous cell carcinoma patients SPAG6 SNVs were seen in only $2 \%$. Homozygous SPAG6 deletions were detected in only $1 \%$ and heterozygous SPAG6 deletions were found in $40 \%$ of these patients. Similar frequencies of SNVs and deletions were found for L1TD1 (Additional file 4: Figure S3).

\section{Frequent loss of SPAG6 and L1TD1 protein expression in NSCLC patients}

To compare SPAG6 and L1TD1 methylation with their protein expression in NSCLC patients, we performed IHC of FFPE TU and NL samples of a subgroup of 35 NSCLC patients. These samples were also analysed for 

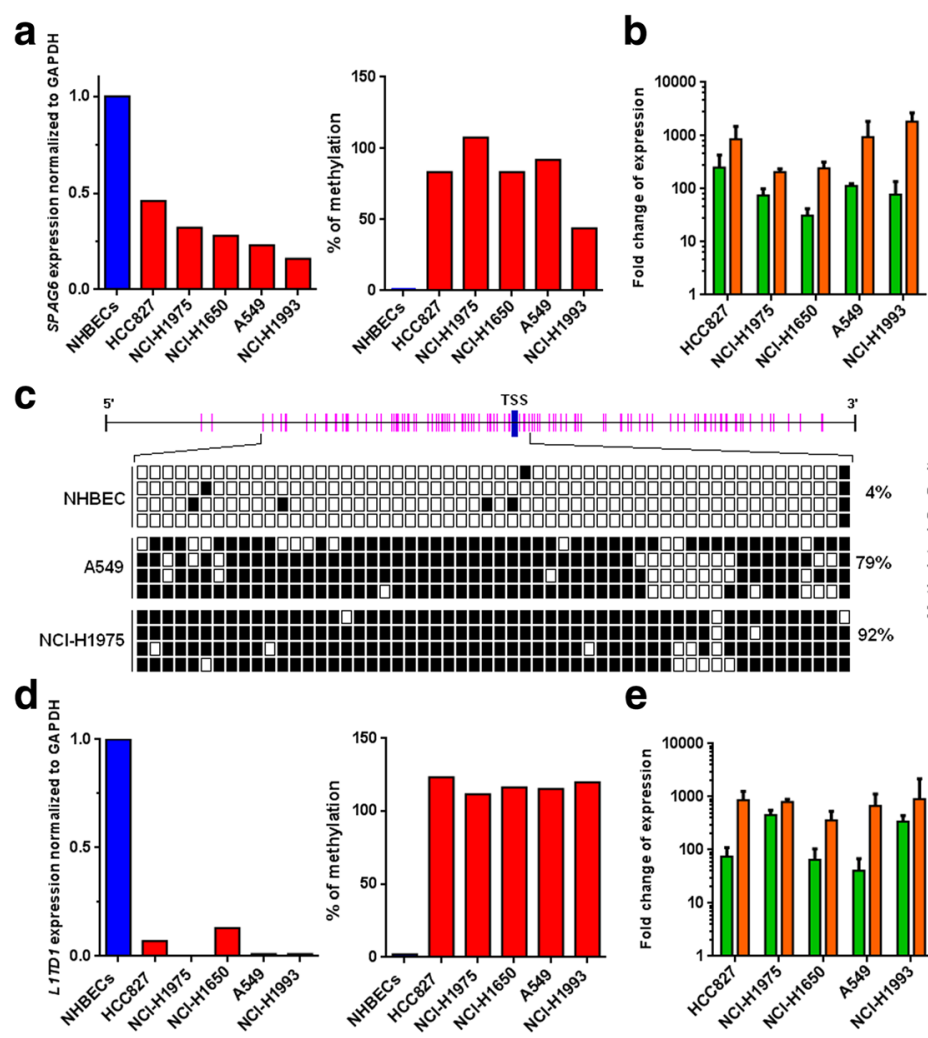

e
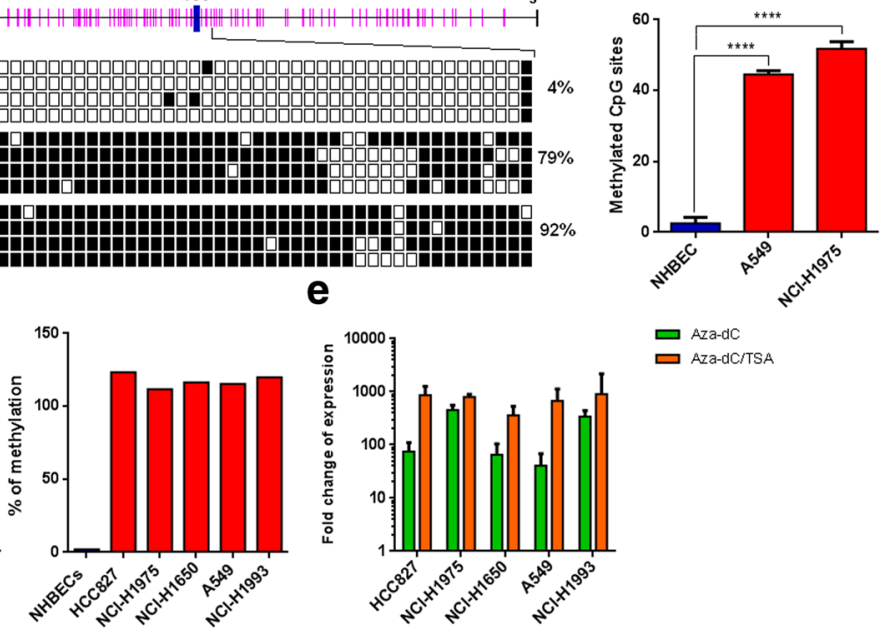

$\square$ Aza-dC

$\square$ Aza-dC/TSA
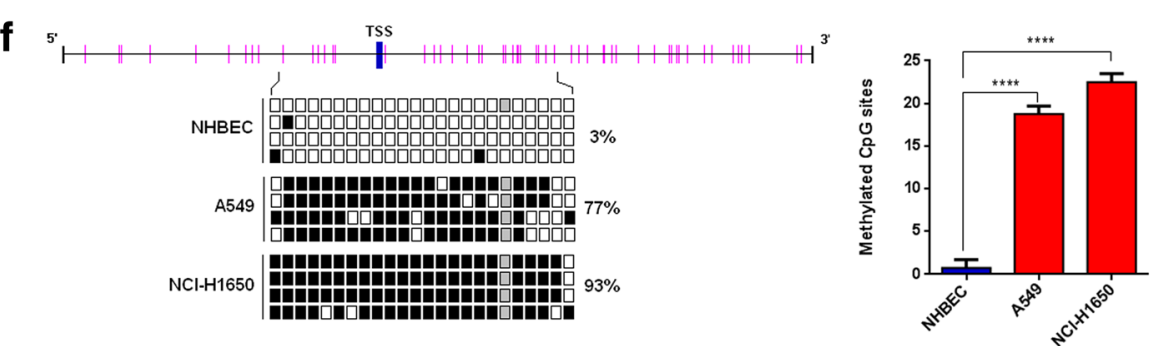

Fig. 2 Expression and methylation analyses of SPAG6 and L1TD1 in NHBECS and in NSCLC cell lines. a By RT-PCR SPAG6 mRNA was found to be downregulated in NSCLC cell lines but not in NHBECS. In contrast, SPAG6 methylation was observed in all NSCLC cell lines with downregulated mRNA expression but not in NHBECs by MS-HRM analysis. b SPAG6 methylated cells were treated with 5-AzadC alone or with a combination of 5AzadC and TSA. RT-PCR analyses revealed upregulation of SPAG6 expression after drug treatment. Fold changes of gene expression in treated cells compared to untreated cells are shown. c Results from BGS of a part of the SPAG6 5' region in NHBECs, A549 and NCI-H1975 cells are shown. 56 CpG sites (indicated as pink bars) were analysed for SPAG6 methylation. TSS, transcription start site, black squares indicate methylated CpG sites, white squares unmethylated CpG sites. Analyses of BGS results of the SPAG6 5' region showed statistically significant differences of methylated CpG sites between NHBCEs and NSCLC cells $\left({ }^{* * *} p<0.0001\right)$. $\mathbf{d}$ L1TD1 mRNA was found to be downregulated in NSCLC cell lines but not in NHBECS by RT-PCR. By MS-HRM analysis L1TD1 methylation was detected in all NSCLC cell lines with downregulated mRNA expression but not in NHBECS. e L1TD1 methylated cells were treated with epigenetically active drugs. RT-PCR analyses revealed upregulation of L1TD1 expression after drug treatment. Fold changes of gene expression in treated cells compared to untreated cells are shown. $\mathbf{f}$ Results from BGS of a part of the L1TD1 5' region in NHBECs, A549 and NCl-H1650 cells are shown. Twenty-four CpG sites were analysed for L1TD1 methylation. TSS, transcription start site, black squares indicate methylated $\mathrm{CpG}$ sites, white squares unmethylated $\mathrm{CpG}$ sites and grey squares intermediate methylation. Analysis of BGS results of the L1TD1 5' region showed statistically significant differences of methylated CpG sites between NHBCEs and NSCLC cells $\left({ }^{* * *} p<0.0001\right)$

SPAG6 and L1TD1 methylation by MS-HRM. SPAG6 and L1TD1 protein expression was observed in bronchial and bronchiolar epithelial cells of NL samples. L1TD1 protein expression was also detected in alveolar epithelial cells. However, no or only weak SPAG6 staining was detected in $60 \%$ and in $40 \%$ of the TU samples, respectively. Moreover, no L1TD1 protein expression was detected in $51 \%$ and only weak staining in $11 \%$ of the TU samples. Moderate or strong staining was seen in $20 \%$ and in $17 \%$ of the TU specimens, respectively. Representative stainings of TU and NL samples are shown in Fig. 4. When we compared SPAG6 and L1TD1 

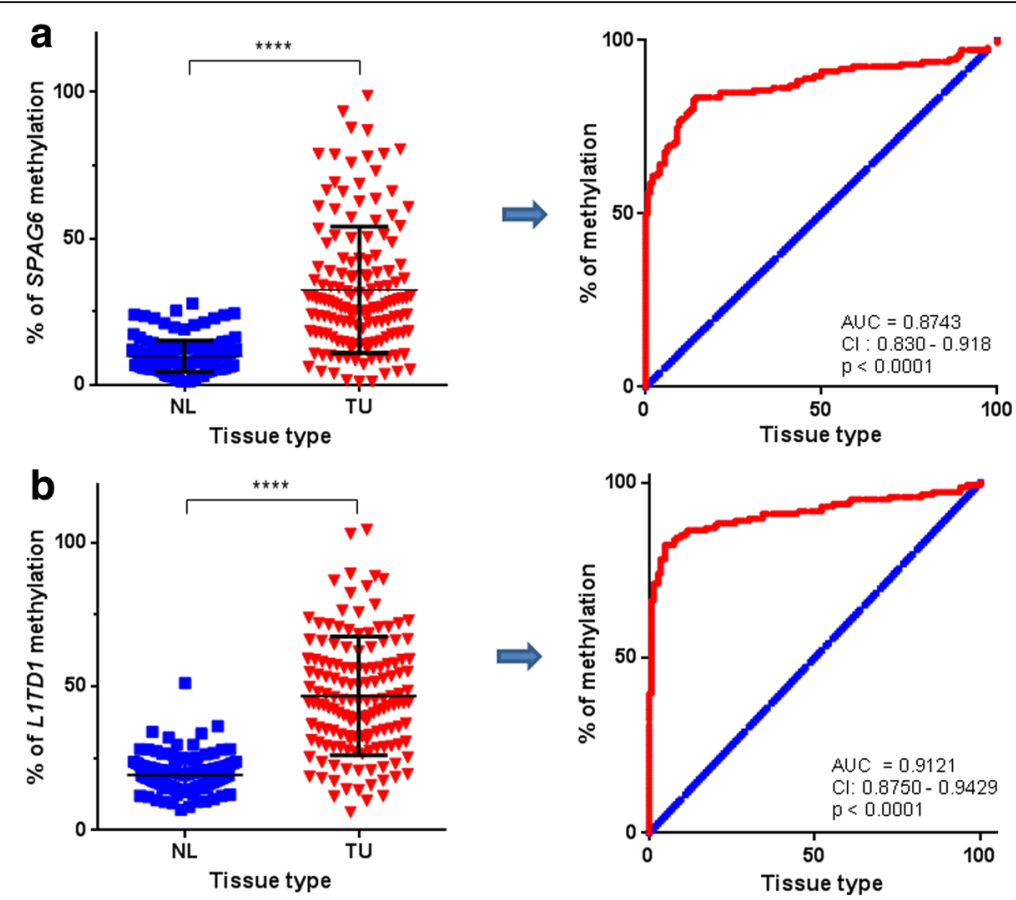

Fig. 3 SPAG6 and L1TD1 methylation in NSCLC patients. Comparison of SPAG6 $\mathbf{a}$ and L1TD1 b methylation percentages in TU and NL samples of 146 NSCLC patients with each dot representing methylation percentages in an individual sample revealed statistically significant differences $\left.{ }^{* * * *} p<0.0001\right)$. ROC curve analyses demonstrated that the extent of SPAG6 $\mathbf{a}$ and L1TD1 $\mathbf{b}$ methylation is able to distinguish between TU and NL samples. Blue line, reference line; red line, methylation of SPAG6; AUC, area under the curve; $\mathrm{Cl}$, confidence interval; TU, primary tumor sample; NL, normal lung tissue sample

methylation with their protein expression in TU samples, we observed downregulated SPAG6 protein expression in $77 \%$ of SPAG6 methylated TU samples and downregulated L1TD1 protein expression in 52\% of L1TD1 methylated TU samples, respectively (Fig. 4c).

Effects of SPAG6 and L1TD1 expression on growth, proliferation, viability and colony-forming abilities of NSCLC cells

To investigate potential tumor cell - growth suppressing properties of L1TD1, we stably transfected NCI-H1975 cells with a pCMV6-L1TD1 expression vector, with a pCMV6-ENTRY control vector as well as with a constructed pCMV6-GFP vector. We found a strong reduction in tumor cell growth of pCMV6-L1TD1 compared to pCMV6-ENTRY transfected cells (Fig. 5a). Overexpression of L1TD1 in pCMV6-L1TD1 transfected cells was confirmed by RT-PCR (Fig. 5b) and IP-WB (Fig. 5c). Moreover, we analysed tumor cell proliferation in a time-dependent manner and observed a reduced proliferation rate of pCMV6-L1TD1 compared to pCMV6ENTRY and to pCMV6-GFP transfected cells (Fig. 5F). In addition, we found a reduced colony-forming ability and tumor cell viability of pCMV6-L1TD1 transfected cells compared to control cells (Fig. 5d, e). Differences in tumor cell viability between pCMV6-L1TD1 and pCMV6-
ENTRY transfected cells were statistically significant after $48(p=0.0064)$ and $72 \mathrm{~h}(p<0.0001)$, while differences between pCMV6-L1TD1 and pCMV6-GFP transfected cells did not reach statistical significance. Similar in vitro experiments were performed for SPAG6. However, no impact of ectopic SPAG6 expression on tumor cell growth, proliferation, viability or colony-forming abilities were seen (data not shown).

\section{L1TD1 reduces tumor growth in vivo}

Because in vitro experiments suggested tumor-cell growth suppressing properties of L1TD1, we additionally performed in vivo studies using immunodeficient mice. Therefore, pCMV6-L1TD1 and pCMV6-ENTRY transfected cells were subcutaneously injected into the ventral flanks of NSG mice. Ten days after injection 4/4 mice had measurable lesions in both flanks. From day 18 after injection on statistically significant differences in tumor volumes between the two groups were observed (Fig. 6a). At day 28 after injection the experiment was terminated and tumors were dissected. Size measurement of dissected tumors confirmed size differences of tumors derived from pCMV6-L1TD1 and from pCMV6-ENTRY transfected cells which were seen during the experiment (Fig. 6b). Expression of L1TD1 in tumors derived from pCMV6- 


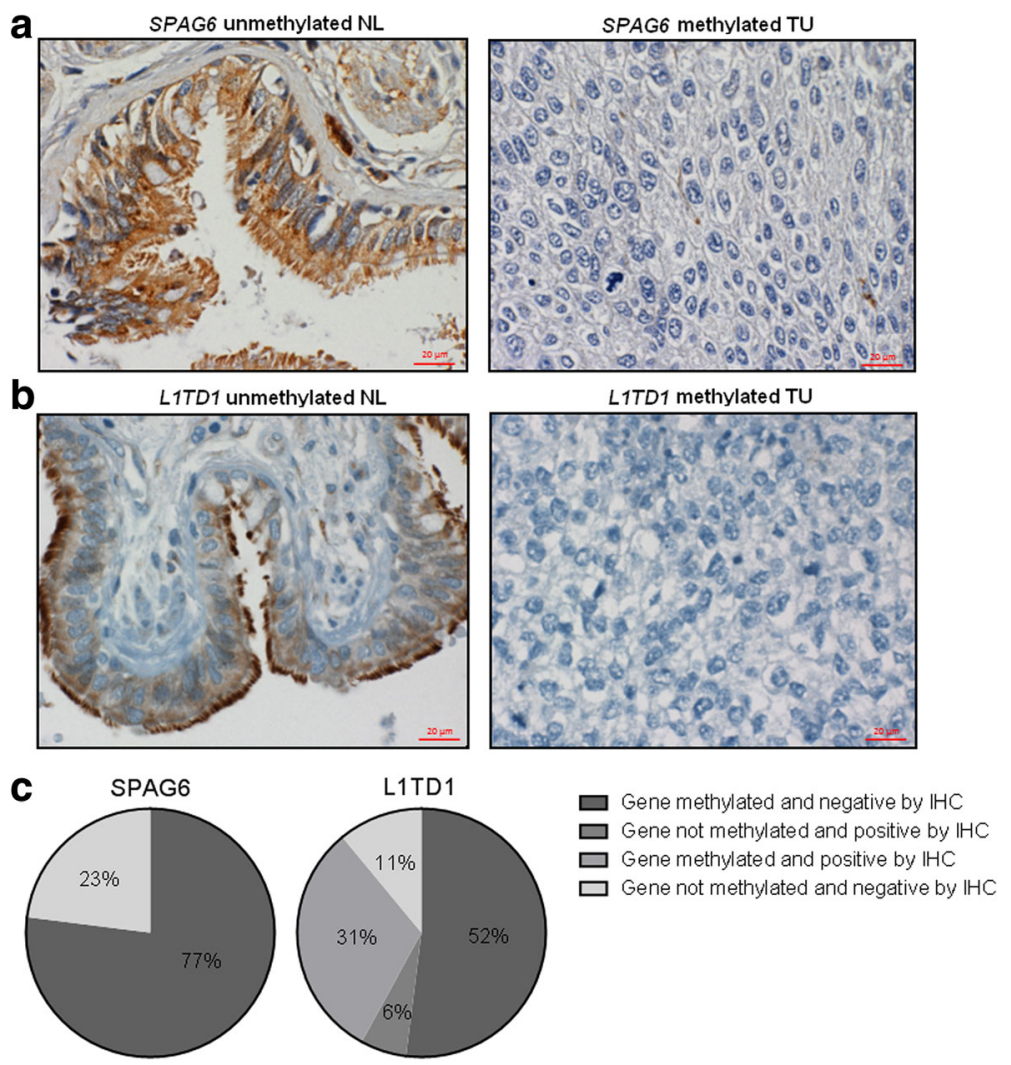

Fig. 4 Protein expression of SPAG6 and L1TD1 in NSCLC patients. Representative IHC stainings of NL and TU samples are shown. In unmethylated NL samples cytoplasmic expression of SPAG6 $\mathbf{a}$ and L1TD1 $\mathbf{b}$ was observed in bronchial and bronchiolar epithelial cells. In SPAG6 a, respectively, L1TD1 b methylated TU samples neither SPAG6 nor L1TD1 expression was observed in tumor cells. $\mathbf{c}$ The comparison between SPAG6 and L1TD1 protein expression and SPAG6 or L1TD1 methylation by T/N methylation ratios and IHC groupings of 35 TU samples is shown

L1TD1 transfected NCI-H1975 cells was confirmed by RT-PCR (Additional file 5: Figure S4).

\section{Discussion}

In a recent study, we performed a genome-wide screen for CpG island methylation in NSCLC patients and identified more than 400 tumor-specifically methylated genes. Two of them, SPAG6 and L1TD1, were selected for detailed investigation of gene expression, genespecific methylation and potential tumor-cell growth suppressing properties in NSCLCs. So far, only little information about a potential impact of these 2 genes on the development of lung cancer was available.

SPAG6 regulates proliferation and differentiation of certain cell types and belongs to the family of CTAs [35, 36]. Normally CTAs are expressed by gametes and trophoblasts but are aberrantly expressed in a variety of tumors [21]. Their transcriptional activity is mainly regulated by epigenetic modifications including methylation and histone acetylation [21, 37]. The role of CTAs in germ line as well as in tumor tissues is poorly understood, however, since germ and cancer cells share certain characteristics including immortalization, invasion and migration, an involvement of CTAs in the development of different tumor types is suggested [37].

L1TD1 is involved in the regulation of self-renewal and pluripotency of human embryonic stem cells. It is highly expressed in medulloblastoma cells where it is associated with cell viability, chemotherapeutic drug resistance and stem cell-like properties [38].

To determine a potential role of SPAG6 and/or L1TD1 in various malignancies, we analysed RNA-seq datasets from the TCGA database. While tumor-specific downregulation of SPAG6 mRNA expression was observed in all tumor types investigated except hepatocellular and prostate carcinomas, downregulated L1TD1 mRNA expression was found in NSCLCs, breast, colorectal and prostate carcinomas but not in head and neck, kidney and hepatocellular carcinomas. Overall, these data suggest that deregulated expression of SPAG6 and L1TD1 may play a role not only in the pathogenesis of NSCLCs but also in tumors of other entities and that expression of these 2 genes differs between certain tumor types.

Besides other mechanisms, methylation is involved in the regulation of transcriptional gene activity $[1,2]$. In NSCLCs, many TSGs are already known which are 


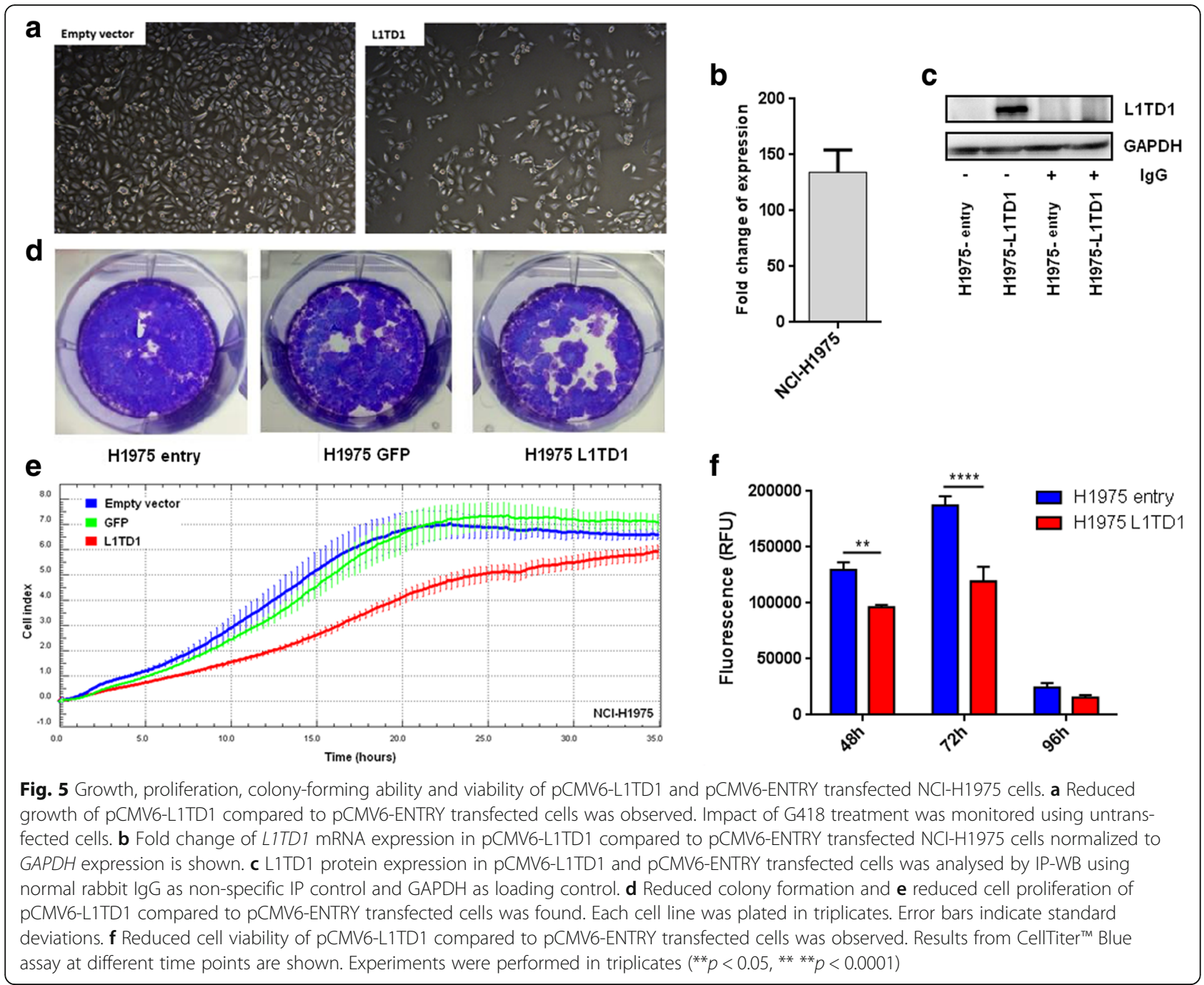

frequently inactivated by methylation $[11,13,15,39]$. To determine if downregulation of SPAG6 and L1TD1 may be caused by methylation, we performed gene-specific approaches to detect mRNA/protein expression and methylation of these genes in NSCLC cell lines and in primary TU and NL samples from NSCLC patients. Indeed, we found a correlation between downregulated SPAG6 and L1TD1 mRNA expression and methylation of these genes in all NSCLC cell lines analysed. Treatment of these cells with epigenetically active drugs which inhibit DNA methyltransferases and histone deacetylases resulted in upregulated expression of both genes. In addition, we found an association between SPAG6 and L1TD1 methylation and loss of SPAG6 and L1TD1 protein expression when we performed IHC of FFPE tissue samples from NSCLC patients. While most of the SPAG6 or L1TD1 methylated TU samples did not express these proteins, not methylated TU samples mostly expressed SPAG6 or L1TD1. However, these results did not reach statistical significance, probably because of the low sample number available for these analyses and the fact that tissue microarrays only represented small parts of the primary tumor samples. Our hypothesis that methylation is one of the mechanisms responsible for inactivation of these genes is further supported by the fact that SNVs and homozygous deletions of SPAG6 and L1TD1 were rarely detected in LUAD and LUSC SNP and in aCGH datasets of NSCLC patients. However, other mechanisms including deregulation of histone modifications and non-coding RNAs may also contribute to the transcriptional regulation of these genes. All these findings indicate that methylation is indeed involved in the transcriptional regulation of SPAG6 and L1TD1 in NSCLCs.

Methylation of certain TSGs in NSCLCs is tumorsubtype specific [40]. While for instance $A P C$ and CDH13 methylation was detected more frequently in lung adenocarcinomas compared to lung squamous cell carcinomas, p16 methylation was detected more frequently in lung squamous cell carcinomas than in lung 

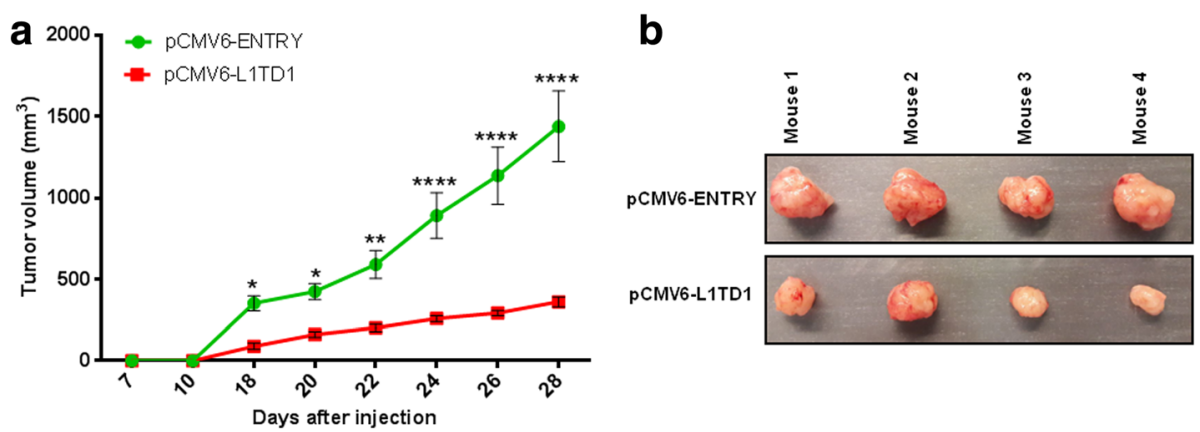

Fig. 6 Xenograft tumor model. a Reduced volumes of xenograft tumors derived from pCMV6-L1TD1 compared to pCMV6-ENTRY transfected NCIH1975 cells were observed and are shown as mean with SEM $\left({ }^{* *} p=0.0078\right.$ respectively $\left.p=0.0075,{ }^{* * *} p=0.0003,{ }^{* * *} p<0.0001\right)$. b Differences in the size of xenograft tumors were confirmed after dissection. Upper panel: xenograft tumors expressing pCMV6-ENTRY, lower panel: xenograft tumors expressing PCMV6-L1TD1

adenocarcinomas [40, 41]. By comparing SPAG6 and L1TD1 methylation with histological classification of our tumor samples, we did not find a difference between the frequencies of methylation of these genes in lung adenocarcinomas or lung squamous cell carcinomas. These observations suggest that methylation of SPAG6 and L1TD1 is a common feature in all histological subtypes of NSCLCs. Methylation of several genes was shown to be associated with shorter survival of NSCLC patients (e.g. APC, CDH1, DAPK or $p 16)$ [13, 42, 43]. In our study, we did not find a correlation between SPAG6 or L1TD1 methylation and OS as well as DFS of NSCLC patients or any other clinico-pathological characteristic of these patients. However, analyses of gene expression microarray data indicate that low SPAG6 expression is associated with a shorter OS of lung squamous cell carcinoma patients and low L1TD1 expression with a shorter OS of lung adenocarcinoma patients. Nevertheless, these findings need to be studied in larger patient cohorts.

TSGs are characterised by a variety of molecular features including frequent $\mathrm{CpG}$ island methylation, downregulated expression and that they are often located in regions of LOH. Since SPAG6 and L1TD1 were found to be frequently methylated and downregulated in NSCLC cells and are located in chromosomal regions where frequent $\mathrm{LOH}$ was observed, we hypothesized that these genes may have tumor-cell growth suppressing properties. Indeed, in vitro experiments showed reduced cell growth, proliferation, viability and colony-forming abilities of pCMV6L1TD1 transfected cells suggesting that it may be a potential TSG in NSCLCs. Because of these encouraging results and to further support our hypothesis that L1TD1 has tumor-cell growth suppressing properties, we additionally performed xenograft experiments to investigate the growth of tumors induced by L1TD1- overexpressing and wildtype NSCLC cells. Interestingly, we observed significantly smaller tumors induced by pCMV6-L1TD1 compared to pCMV6-ENTRY transfected NCI-H1975 cells indicating that L1TD1 indeed has tumor-cell growth suppressing properties. Based on the literature this is the first report which describes a potential impact of L1TD1 expression in the pathogenesis of NSCLCs. However, further studies are necessary to elucidate molecular mechanisms affected by L1TD1 in NSCLC cells.

Although it was reported that SPAG6 is involved in proliferation and differentiation of neuronal progenitor cells, in our in vitro studies ectopic SPAG6 expression did not affect the behavior of NSCLC cells $[35,36]$. Since no reports are currently available about a potential role of SPAG6 in malignant diseases further studies are necessary to determine celltype specific functions of SPAG6 and to elucidate if besides tumor-specific methylation other functions of SPAG6 may be involved in the pathogenesis of NSCLCs.

\section{Conclusion}

Our results demonstrate that tumor-specific methylation of SPAG6 and L1TD1 is a frequently occurring event in NSCLCs and they suggest that methylation plays an important role in the transcriptional regulation of these genes. Protein expression of both genes was frequently downregulated in primary NSCLCs. In addition, or findings indicate that methylation of these genes may be of relevance not only in NSCLCs but also in other malignancies. Moreover, in vitro and in vivo experiments showed that L1TD1 has tumor-cell growth suppressing properties in NSCLC cells. Taken together we identified methylation as a potential mechanism for frequent downregulation of SPAG6 and L1TD1 in NSCLCs and suggest a putative role of L1TD1 in tumor cell development. 


\section{Additional files}

Additional file 1: Table S1. Description of NSCLC cell lines used in this study. Information about histology, origin and disease stage of donors was obtained from ATCC catalogue (https://www.lgcstandards-atcc.org). EGFR, KRAS and TP53 mutational status and MET amplification according to supplementary references (1-3). *activating EGFR mutation in exon 19 (E746-E749 del), **activating EGFR mutation in exon 21 (L858R). N/A, not available; wt, wildtype; mut, mutated. Table S2. Clinico-pathological characteristics of 983 NSCLC patients. Overview of gender, histology, stage of disease and ethnicity of NSCLC patients obtained from TCGA database and used for mutation and copy number changes analyses of SPAG6 and L1TD1 is shown. ADC, adenocarcinoma; SCC, squamous cell carcinoma. Clinical data based on Caleydo software version 16/04/14. Table S3. Primer sequences. Summary of oligonucleotide sequences used for mRNA expression, MS-HRM, BGS analyses and construction of pCMV6GFP expression vector. $Y$, random integration of $C$ or $T$ in fwd primer; $R$, random integration of $\mathrm{G}$ or $\mathrm{A}$ in rev primer. Table S4. Methylation of SPAG6 and L1TD1 in tumor cells of other tumor types. *Morphology, histology and origin of cell lines according to ATCC catalogue (https:// www.lgcstandards-atcc.org). Percentage of methylation was calculated as described previously (4). (DOCX $33 \mathrm{~kb}$ )

Additional file 2: Figure S1. SPAG6 and LITD1 mRNA expression in different datasets of TCGA database. SPAG6 and L1TD1 mRNA expression was analysed using IlluminaHiSeq RNAseq data from TCGA database. Datasets LUAD and LUSC (lung), BRCA (breast), COADREAD (colorectal), HNSC (head and neck), KIRC (kidney), LIHC (liver) and PRAD (prostate) were analysed. Normalized log2 mRNA expression values are shown. Each dot represents a single sample. (TIF $176 \mathrm{~kb}$ )

Additional file 3: Figure S2. Impact of SPAG6 and L1TD1 mRNA expression on OS of NSCLC patients. (A) A shorter OS of squamous cell carcinoma patients with low SPAG6 mRNA expression $(N=155)$ compared to high SPAG6 mRNA expression ( $N=267$ ) was observed. (B) Adenocarcinoma patients with low L1TD1 mRNA expression $(\mathrm{N}=138)$ showed a shorter OS compared to adenocarcinoma patients with high L1TD1 mRNA expression ( $N=350)$. Gene expression microarray datasets (Affymetrix IDs 210032_s_at and 219955_at) were analysed and Kaplan-Meier plots were generated using all datasets and default settings of KM plotter. The cut-off values for "low" and "high" SPAG6 and L1TD1 mRNA expression were automatically defined by KM plotter software (Version 2013). (TIF 50 kb)

Additional file 4: Figure S3. SPAG6 and LITD1 SNVs and deletions in NSCLC patients. TCGA LUAD and LUSC datasets were analysed with Caleydo software (version April 2014). Mutation of TP53 was used to demonstrate reliability of TCGA data analysis. ADC, adenocarcinoma patients; SCC, squamous carcinoma patients. (TIF 90 kb)

Additional file 5: Figure S4. L1TD1 mRNA expression in xenograft tumors. Expression of L1TD1 in 4 xenografts derived from pCMV6-L1TD1 transfected NCl-H1975 cells was confirmed by RT-PCR. GAPDH was used as housekeeping gene to normalize mRNA expression of L1TD1. (TIF $17 \mathrm{~kb}$ )

\section{Abbreviations}

5-AzaC: 5-azacytidine; 5-AzadC: 5-aza-2'-deoxycytidine; aCGH: Microarray Comperative Genomic Hybridization; BGS: Bisulfite genomic sequencing; BRCA: Breast invasive carcinoma; COADREAD: Colon and rectum adenocarcinoma; CTA: Cancer-testis antigen; DFS: Disease free survival; DNMT: DNA methyltransferases; FFPE: Formalin-fixed, paraffin embedded samples; HDAC: Histone deacetylase; HNSC: Head and neck squamous cell carcinoma; IHC: Immunohistochemistry; IP-WB: Immunoprecipitation and Western blot; KIRC: Kidney clear cell carcinoma; LIHC: Liver hepatocellular carcinoma; LUAD: Lung adenocarcinoma dataset; LUSC: Lung squamous cell carcinoma dataset; MBD: methyl-CpG binding domain proteins; MSHRM: Methylation-sensitive high resolution melting analysis; NHBEC: Normal human bronchial epithelial cell; NL: Corresponding non-malignant lung tissue samples; NSCLC: Non-small cell lung cancer; NSG mice: NSG JAX (NOD.Cg-Prkdc $\left.c^{\text {scid } / / 2 g^{t m} / W j l} / S z J\right)$ mice; OS: Overall survival; PRAD: Prostate adenocarcinoma; RNA-seq: IlluminaHiSeq RNA-sequencing; ROC: Receiver operating characteristic; RT-PCR: Real-time reverse transcription PCR; SNV: Single nucleotide variants; TCGA: "The Cancer Genome Atlas"; TSA: Trichostatin A; TSG: Tumor suppressor gene; TU: Primary tumor samples

\section{Acknowledgement}

Not applicable.

\section{Funding}

This work was funded by the Austrian Science Fund (FWF) through projects F4709-B20 and P24130 to Sabine Zöchbauer-Müller, by Clinical Research Grants of the Austrian Society of Hematology and Oncology (ÖGHO) to Corinna Altenberger and to Gerwin Heller and by a Research Grant of the "Initiative Krebsforschung" of the Medical University of Vienna to Gerwin Heller.

\section{Availability of data and materials}

The datasets analysed during this study are available in the TCGA database (http://cancergenome.nih.gov), Cancer Browser (https://genome-cancer.ucsc.edu) and cBioPortal for Cancer Genomics (http://www.cbioportal.org/).

\section{Authors' contributions}

The contributions of the authors are: Study design and concept: $\mathrm{CA}, \mathrm{GH}$, SZM; Data acquisition: CA, GH, BZ, ET, MM, TT and PH; Data analysis and interpretation: $\mathrm{CA}, \mathrm{GH}, \mathrm{LM}$; Collection of clinical data and sample disposal: GL, AEP, BD, BMA and WK; Manuscript preparation: CA and SZM; Manuscript review: CA, GH, BZ, ET, MM, TT, LM, PH, GL, AEP, BD, BA, WK, CCZ and SZM. All authors read and approved the final manuscript.

\section{Competing interests}

The authors declare that they have no competing interests.

\section{Consent for publication}

Not applicable.

\section{Ethics approval}

The study was approved by the Ethics Committee of the Medical University of Vienna. All animal experiments were approved by and carried out according to guidelines from the Austrian Federal Ministry of Science, Research and Economy and the Animal Ethics Committee of the Medical University of Vienna.

\section{Author details}

${ }^{1}$ Department of Medicine I, Clinical Division of Oncology, Medical University of Vienna, Währinger Gürtel 18-20, 1090 Vienna, Austria. ${ }^{2}$ Comprehensive Cancer Center, Medical University of Vienna, Vienna, Austria. ${ }^{3}$ Department of Pathology, Medical University of Vienna, Vienna, Austria. ${ }^{4}$ Department of Medicine I, Institute of Cancer Research, Medical University of Vienna, Vienna, Austria. ${ }^{5}$ Division of Thoracic Surgery, Medical University of Vienna, Vienna, Austria. ${ }^{6}$ Department of Thoracic Oncology and Tumor Biology, National Koranyi Institute of Pulmonology, Budapest, Hungary. 'Department of Thoracic Surgery, Semmelweis University and National Institute of Oncology, Budapest, Hungary. ${ }^{8}$ Department of Respiratory and Critical Care Medicine and Ludwig Boltzmann Institute for COPD, Otto-Wagner Hospital, Vienna, Austria.

Received: 29 July 2016 Accepted: 7 December 2016 Published online: 05 January 2017

\section{References}

1. Jones PL, Veenstra GJ, Wade PA, Vermaak D, Kass SU, Landsberger N, et al. Methylated DNA and MeCP2 recruit histone deacetylase to repress transcription. Nat Genet. 1998;19:187-91.

2. Wade PA. Methyl CpG-binding proteins and transcriptional repression. Bioessays. 2001:23:1131-7.

3. Esteller M. Epigenetics in cancer. N Engl J Med. 2008;358:1148-59.

4. Kulis M, Esteller M. DNA methylation and cancer. Adv Genet. 2010;70:27-56.

5. Baylin SB. DNA methylation and gene silencing in cancer. Nat Clin Pract Oncol. 2005;2 Suppl 1:S4-11.

6. Robertson KD. DNA methylation and human disease. Nat Rev Genet 2005;6:597-610

7. Bird A. DNA methylation patterns and epigenetic memory. Genes Dev. 2002;16:6-21.

8. Jones PA. Functions of DNA methylation: islands, start sites, gene bodies and beyond. Nat Rev Genet. 2012;13:484-92.

9. Bird AP. CpG-rich islands and the function of DNA methylation. Nature. 1986;321:209-13. 
10. Cameron EE, Bachman KE, Myohanen S, Herman JG, Baylin SB. Synergy of demethylation and histone deacetylase inhibition in the re-expression of genes silenced in cancer. Nat Genet. 1999;21:103-7.

11. Heller G, Altenberger C, Schmid B, Marhold M, Tomasich E, Ziegler B, et al. DNA methylation transcriptionally regulates the putative tumor cell growth suppressor ZNF677 in non-small cell lung cancers. Oncotarget. 2015:6:394-408.

12. Heller G, Babinsky VN, Ziegler B, Weinzierl M, Noll C, Altenberger C, et al. Genome-wide $\mathrm{CpG}$ island methylation analyses in non-small cell lung cancer patients. Carcinogenesis. 2013;34:513-21.

13. Zöchbauer-Müller S, Fong KM, Virmani AK, Geradts J, Gazdar AF, Minna JD Aberrant promoter methylation of multiple genes in non-small cell lung cancers. Cancer Res. 2001;61:249-55.

14. Heller G, Fong KM, Girard L, Seidl S, End-Pfützenreuter A, Lang G, et al. Expression and methylation pattern of TSLC1 cascade genes in lung carcinomas. Oncogene. 2006;25:959-68.

15. Sandoval J, Mendez-Gonzalez J, Nadal E, Chen G, Carmona FJ, Sayols S, et al. A prognostic DNA methylation signature for stage I non-small-cell lung cancer. J Clin Oncol. 2013;31:4140-7.

16. Sanchez-Palencia A, Gomez-Morales M, Gomez-Capilla JA, Pedraza V, Boyero $L$, Rosell $R$, et al. Gene expression profiling reveals novel biomarkers in nonsmall cell lung cancer. Int J Cancer. 2011;129:355-64.

17. Hou J, Aerts J, den Hamer B, van ljcken W, den Bakker M, Riegman P, et al. Gene expression-based classification of non-small cell lung carcinomas and survival prediction. PLoS One. 2010;5:e10312.

18. Yang B, Wang L, Luo X, Chen L, Yang Z, Liu L. SPAG6 silencing inhibits the growth of the malignant myeloid cell lines SKM-1 and K562 via activating p53 and caspase activation-dependent apoptosis. Int J Oncol. 2015;46:649-56.

19. Silina K, Zayakin P, Kalnina Z, Ivanova L, Meistere I, Endzelins E, et al. Sperm-associated antigens as targets for cancer immunotherapy: expression pattern and humoral immune response in cancer patients. J Immunother. 2011;34:28-44.

20. Lonergan KM, Chari R, Deleeuw RJ, Shadeo A, Chi B, Tsao MS, et al. Identification of novel lung genes in bronchial epithelium by serial analysis of gene expression. Am J Respir Cell Mol Biol. 2006;35:651-61.

21. Fratta E, Coral S, Covre A, Parisi G, Colizzi F, Danielli R, et al. The biology of cancer testis antigens: putative function, regulation and therapeutic potential. Mol Oncol. 2011;5:164-82.

22. Fong KM, Kida Y, Zimmerman PV, Smith PJ. MYCL genotypes and loss of heterozygosity in non-small-cell lung cancer. $\mathrm{Br} J$ Cancer. 1996;74:1975-8.

23. Narva E, Rahkonen N, Emani MR, Lund R, Pursiheimo JP, Nasti J, et al. RNAbinding protein L1TD1 interacts with LIN28 via RNA and is required for human embryonic stem cell self-renewal and cancer cell proliferation. Stem Cells. 2012;30:452-60.

24. Goldman M, Craft B, Swatloski T, Ellrott K, Cline M, Diekhans M, et al. The UCSC Cancer Genomics Browser: update 2013. Nucleic Acids Res. 2013;41:D949-54.

25. Cerami E, Gao J, Dogrusoz U, Gross BE, Sumer SO, Aksoy BA, et al. The cBio cancer genomics portal: an open platform for exploring multidimensional cancer genomics data. Cancer Discov. 2012;2:401-4.

26. Gao J, Aksoy BA, Dogrusoz U, Dresdner G, Gross B, Sumer SO, et al. Integrative analysis of complex cancer genomics and clinical profiles using the cBioPortal. Sci Signal. 2013;6:11.

27. Cancer Genome Atlas Research Network. Comprehensive genomic characterization of squamous cell lung cancers. Nature. 2012;489:519-25.

28. Cancer Genome Atlas Research Network. Comprehensive molecular profiling of lung adenocarcinoma. Nature. 511:543-50.

29. Streit M, Lex A, Kalkusch M, Zatloukal K, Schmalstieg D. Caleydo: connecting pathways and gene expression. Bioinformatics. 2009;25:2760-1.

30. Heller G, Schmidt WM, Ziegler B, Holzer S, Müllauer L, Bilban M, et al. Genome-wide transcriptional response to 5-aza-2'-deoxycytidine and trichostatin a in multiple myeloma cells. Cancer Res. 2008;68:44-54.

31. Heller G, Weinzierl M, Noll C, Babinsky V, Ziegler B, Altenberger C, et al. Genome-wide microRNA expression profiling identifies miR-9-3 and miR-193a as targets for DNA methylation in non-small cell lung cancers. Clin Cancer Res. 2012;18:1619-29.

32. Schmittgen TD, Livak KJ. Analyzing real-time PCR data by the comparative C(T) method. Nat Protoc. 2008;3:1101-8

33. Tomayko MM, Reynolds CP. Determination of subcutaneous tumor size in athymic (nude) mice. Cancer Chemother Pharmacol. 1989;24:148-54.
34. Gyorffy B, Surowiak P, Budczies J, Lanczky A. Online survival analysis software to assess the prognostic value of biomarkers using transcriptomic data in non-small-cell lung cancer. PLoS One. 2013;8:e82241.

35. Hu X, Yan R, Cheng X, Song L, Zhang W, Li K, et al. The function of spermassociated antigen 6 in neuronal proliferation and differentiation. J Mol Histol. 2016:47:531-40.

36. Li W, Mukherjee A, Wu J, Zhang L, Teves ME, Li H, et al. Sperm Associated Antigen 6 (SPAG6) regulates fibroblast cell growth, morphology, migration and ciliogenesis. Sci Rep. 2015:5:16506.

37. Simpson AJ, Caballero OL, Jungbluth A, Chen YT, Old LJ. Cancer/testis antigens, gametogenesis and cancer. Nat Rev Cancer. 2005:5:615-25.

38. Santos MC, Silva PB, Rodini CO, Furukawa G, Marco Antonio DS, ZanottoFilho A, et al. Embryonic Stem Cell-Related Protein L1TD1 is required for cell viability, neurosphere formation, and chemoresistance in medulloblastoma. Stem Cells Dev. 2015;24:2700-8.

39. Zöchbauer-Müller S, Minna JD, Gazdar AF. Aberrant DNA methylation in lung cancer: biological and clinical implications. Oncologist. 2002;7:451-7.

40. Toyooka S, Toyooka KO, Maruyama R, Virmani AK, Girard L, Miyajima K, et al. DNA methylation profiles of lung tumors. Mol Cancer Therapeutics. 2001:1:61-7.

41. Heller G, Zielinski CC, Zöchbauer-Müller S. Lung cancer: from single-gene methylation to methylome profiling. Cancer Metastasis Rev. 2010;29:95-107.

42. Esteller M, Corn PG, Baylin SB, Herman JG. A gene hypermethylation profile of human cancer. Cancer Res. 2001:61:3225-9.

43. Toyooka S, Toyooka KO, Miyajima K, Reddy JL, Toyota M, Sathyanarayana UG, et al. Epigenetic down-regulation of death-associated protein kinase in lung cancers. Clin Cancer Res. 2003;9:3034-41.

\section{Submit your next manuscript to BioMed Central and we will help you at every step:}

- We accept pre-submission inquiries

- Our selector tool helps you to find the most relevant journal

- We provide round the clock customer support

- Convenient online submission

- Thorough peer review

- Inclusion in PubMed and all major indexing services

- Maximum visibility for your research

Submit your manuscript at www.biomedcentral.com/submit
) Biomed Central 